Frank Lammert, Petra Lynen Jansen, Markus M. Lerch (Hrsg.)

Weissbuch Gastroenterologie 2020/2021 

Frank Lammert, Petra Lynen Jansen, Markus M. Lerch (Hrsg.)

\section{Weissbuch \\ Gastroenterologie \\ 2020/2021}

Erkrankungen des Magen-Darm-Traktes, der Leber und der Bauchspeicheldrüse Gegenwart und Zukunft

\section{DE GRUYTER}


Herausgeber

Prof. Dr. Dipl.-Kfm. Dipl.-Volksw. Frank Lammert

Klinik für Innere Medizin II

Universitätsklinikum des Saarlandes

Kirrberger Str. 100

66421 Homburg

PD Dr. Petra Lynen Jansen

DGVS - Deutsche Gesellschaft für

Gastroenterologie, Verdauungs- und

Stoffwechselkrankheiten - Gastro Haus

Olivaer Platz 7

10707 Berlin
Prof. Dr. Markus M. Lerch

Klinik für Innere Medizin A

Universitätsmedizin Greifswald

Ferdinand-Sauerbruch-Str.

17475 Greifswald

ISBN: 978-3-11-064232-2

e-ISBN (PDF): 978-3-11-064251-3

e-ISBN (EPUB): 978-3-11-064234-6

\section{(cC) BY-NC-ND}

Dieses Werk ist lizenziert unter der Creative Commons Attribution-NonCommercial-NoDerivatives 4.0 Lizenz. Weitere Informationen finden Sie unter http://creativecommons.org/licenses/by-nc-nd/4.0/.

Library of Congress Control Number: 2019940007

\section{Bibliografische Information der Deutschen Nationalbibliothek}

Die Deutsche Nationalbibliothek verzeichnet diese Publikation in der Deutschen Nationalbibliographie; detaillierte bibliografische Daten sind im Internet über http://dnb.d-nb.de abrufbar.

Der Verlag hat für die Wiedergabe aller in diesem Buch enthaltenen Informationen mit den Autoren große Mühe darauf verwandt, diese Angaben genau entsprechend dem Wissensstand bei Fertigstellung des Werkes abzudrucken. Trotz sorgfältiger Manuskriptherstellung und Korrektur des Satzes können Fehler nicht ganz ausgeschlossen werden. Autoren und Verlag übernehmen infolgedessen keine Verantwortung und keine daraus folgende oder sonstige Haftung, die auf irgendeine Art aus der Benutzung der in dem Werk enthaltenen Informationen oder Teilen davon entsteht.

Die Wiedergabe der Gebrauchsnamen, Handelsnamen, Warenbezeichnungen und dergleichen in diesem Buch berechtigt nicht zu der Annahme, dass solche Namen ohne weiteres von jedermann benutzt werden dürfen. Vielmehr handelt es sich häufig um gesetzlich geschützte, eingetragene Warenzeichen, auch wenn sie nicht eigens als solche gekennzeichnet sind.

(C) 2019 Frank Lammert, Petra Lynen Jansen, Markus M. Lerch, publiziert von Walter de Gruyter $\mathrm{GmbH}$, Berlin/Boston.

Dieses Buch ist als Open-Access-Publikation verfügbar über www.degruyter.com.

Einbandabbildung: Grafik DGVS

Satz/Datenkonvertierung: L42 AG, Berlin

Druck und Bindung: CPI Books GmbH, Leck

www.degruyter.com 